\title{
The Polymerization of Formaldazine. I. The Nature of Formaldazine
}

\author{
Mikiharu Kamachi and Shunsuke MURAhashi \\ Department of Polymer Science, Faculty of Science, \\ Osaka University, Toyonaka, Osaka, 560 Japan.
}

(Received November 5, 1973)

\begin{abstract}
The nature of formaldazine was studied on the basis of the data from various spectroscopies and compared with that of butadiene, its hydrocarbon analog. The UV and far-infrared spectra showed that the delocalization of $\pi$-electrons of formaldazine is less than that of butadiene. The ionization potential of formaldazine was estimated by mass spectroscopy. It is smaller than those of butadiene and triethylamine and is larger than that of dimethylhydrazine. The first ionization comes from the lone pair of the nitrogen, because the unpaired electron has a coupling only with the nitrogen nucleus in the ESR spectrum of the ion salt, which was obtained by the reaction of formaldazine with chloranil. The half wave potential was determined by means of an extrapolation of the Taft's plot of the half wave potentials of various $\alpha, \omega$-dialkylaldehyde azines $(\mathrm{R}-\mathrm{CH}=\mathrm{N}-\mathrm{N}=\mathrm{CH}-\mathrm{R})$ and used as a measure of their electron affinities. Formaldazine was found to have a larger electron affinity than acrylonitrile and butadiene. In conclusion, formaldazine is a compound which has both electron-donating and electron-accepting properties.
\end{abstract}

KEY WORDS Monomer / Formaldazine / $\pi$-Electron / Delocalization / Bond Cleavage / Radical / Ion Salt / Ionization Potential / Electron Affinity /

Formaldazine is the simplest of the azine derivatives and is a particularly interesting compound because it is an analog of butadiene. It was first synthesized by Neureiter in $1959 .{ }^{1}$ Some physical properties have been examined in order to characterize this compound; however, no detailed study on the nature of the azine bond has been reported. As for chemical reactions, the addition reaction of hydrogen sulfide ${ }^{1}$ and the photolysis of formaldazine $e^{2,3}$ have been reported. The high spontaneous polymerizability of formaldazine may have restricted such studies.

The present authors found that formaldazine could be stored above room temperature in some organic solvents. ${ }^{4}$ Investigation about the chemical reactivity and the polymerizability of this compound have been undertaken. ${ }^{4,5}$ But the nature of the azine bond, especially the $\pi$-electron delocalization, must be studied in order to understand sufficiently its chemical reactivity.

In this paper, the nature of the azine bond has been clarified on the basis of data from various spectroscopies. The $\pi$-electron delocali- zation in formaldazine was compared with that in butadiene. The results obtained were explained by the Hückel molecular orbital theory.

\section{EXPERIMENTAL}

\section{Materials}

Aqueous formaldehyde and hydrazine hydrate were commercially obtained and used without purification. Formaldazine was synthesized by means of a modification of Neureiter's procedure. ${ }^{1}$ It was purified by repeated low temperature distillation in a high vacuum system, $\mathrm{mp}-47-$ $-48^{\circ} \mathrm{C}$, (Yield $15 \%$ ). Acetaldazine (bp $95.5^{\circ} \mathrm{C}$ ), propionaldazine (bp $145-146^{\circ} \mathrm{C}$ ), $n$-butyraldazine (bp $183^{\circ} \mathrm{C}$ ), and isobutyraldazine (bp $63^{\circ} \mathrm{C} /$ $14 \mathrm{mmHg}$ ) were synthesized from hydrazine hydrate and the corresponding aldehydes. ${ }^{6}$ They were purified by reduced-pressure distillation through a Widmer column under nitrogen.

Trimethylacetaldazine $\left(\mathrm{mp} 79^{\circ} \mathrm{C}\right)$ was synthesized from hydrazine hydrate and Trimethylacetaldehyde, which was previously prepared from ethyl 
formate and $t$-butylmagnesium bromide, ${ }^{7}$ and was purified by sublimation on a high vacuum system. Dioxane and tetrahydrofuran were purified by the standard methods ${ }^{8}$ and stored over sodium. Before use, they were distilled in a vacuum system. Butadiene (supplied from Japan Synthetic Rubber Co.) which had been dried over molecular sieves was mixed with solventfree $\mathrm{BuLi}$, transferred onto sodium, and then distilled into a glass ampoule.

Chloranil was purified by two recrystallizations from dry benzene and by sublimation.

\section{Measurement}

The IR spectra were measured in the gas state on a Hitachi Model EPI-2 spectrometer using a 10-cm cell. The far-IR spectra were measured in the gas state on a Hitachi Model FIS-1 farinfrared spectrometer using a $10-\mathrm{cm}$ cell. UV spectra were taken in the gas state or in THF solution on a Hitachi Model EPS-2 spectrometer. NMR spectra were taken on a Varian A-60 spectrometer equipped with a variable temperature probe and controller. ESR spectra were measured using an $\mathrm{X}$-band spectrometer made by Kuwata. A 30-\% THF solution of chloranil $(0.12 \mathrm{~g})$ was prepared in an ESR tube. Formaldazine $(0.1 \mathrm{~m} l)$ was added to it by using a high vacuum line. The reaction system was allowed to stand for 5 minutes at $-78^{\circ} \mathrm{C}$, became dark green, and was cooled again to $-196^{\circ} \mathrm{C}$. Its ESR spectrum was measured at $-196^{\circ} \mathrm{C}$ (frozen state). Mass spectra were obtained on a Hitachi Model HRU-10z mass spectrometer.

\section{Polarography}

The polarographic measurement was conducted on a Yanagimoto Model PA-102. As a supporting electrolyte, an $0.04-N$ solution of tetraethylammonium iodide in a $1: 1$ volume ratio mixture of dioxane and water was used.

\section{RESULTS}

The IR spectrum is consistent with that of previous reports. ${ }^{1,2}$ The far-IR spectrum is shown in Figure 1. It is reasonable that $92 \mathrm{~cm}^{-1}$ is assigned to the band of torsional motion around the central single bond, on the basis of the presence of the $Q$-branch in this band and the data concerning the torsion of similar compounds as shown in Table I. ${ }^{9}$

The UV spectrum of formaldazine is shown in Figure 2.

The energy-level diagram suggests that there should be two $n-\pi^{*}$ transitions and one $\pi-\pi^{*}$ transition in the region from 190 to $600 \mathrm{~nm}^{10}$ With the change from gas to THF solution, $203 \mathrm{~nm}$ shifted only slightly, while 228 and $283 \mathrm{~nm}$ shifted and coalesced into a broad absorption band. The absorption at $203 \mathrm{~nm}$ is attributed to the $\pi-\pi^{*}$ transition $\left(\varepsilon=10^{3}-10^{4}\right)$ and that at 228 and $283 \mathrm{~nm}$ to the $n-\pi^{*}$ transitions, by means of the dependency of the absorption bands on the solvent and their approximate $\varepsilon$ values. $\left(\varepsilon=10^{2}-10^{3}\right)$

The NMR spectra of formaldazine are shown in Figure 3. These spectra correspond to those of an $\mathrm{AB}$ system, but are not symmetric with respect to the center of the spectra. This is possibly due to the difference in the interaction of each proton with the nitrogen nuclei. The peaks of the NMR spectra became broader as the

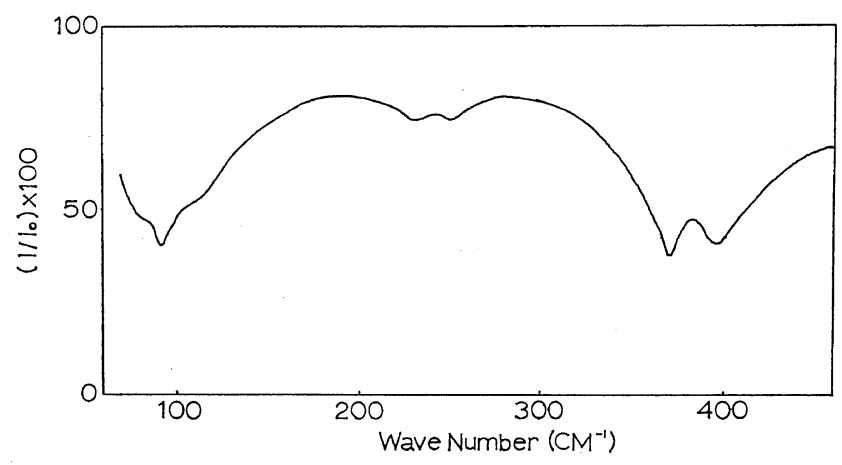

Figure 1. Far-infrared spectrum of formaldazine. Gas state at room temperature. 


\section{Polymerization of Formaldazine}

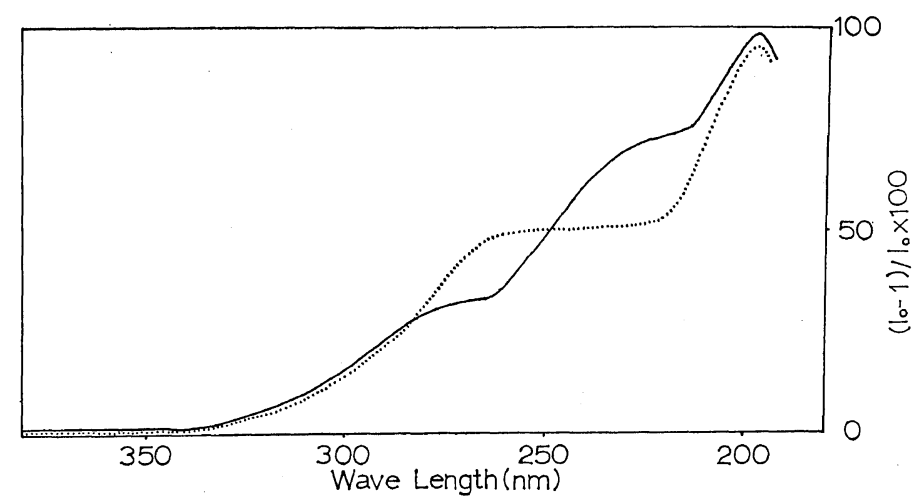

Figure 2. UV spectrum of formaldazine: -, gas; ---, THF solution.

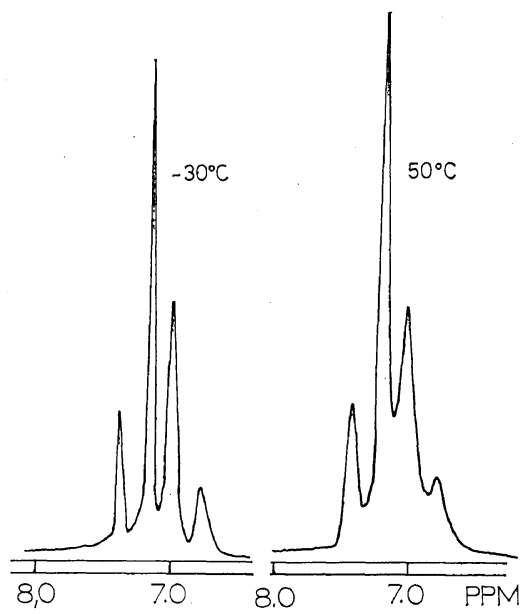

Figure 3. NMR spectra of formaldazine: THF solution, $60 \mathrm{MHz}$. temperature of the measurement was raised. This suggests that the rotation around the central single bond becomes more rapid at higher temperatures.

The mass spectra of this compound are taken at various electron impact potentials. No peak was found in the region above $m / e$ 56, except the isotope peak. The dependency of the ionic current of the parent ion on the electron impact potential was studied and the approximate ionization potential for this compound was determined from the graph of the ionic current against the electron impact potential (Figure 4).

The absolute accuracy of the value cannot be relied upon because there was no calibration of the instrument. To check the accuracy of the observed value, the ionization potential of triethylamine determined by the same method

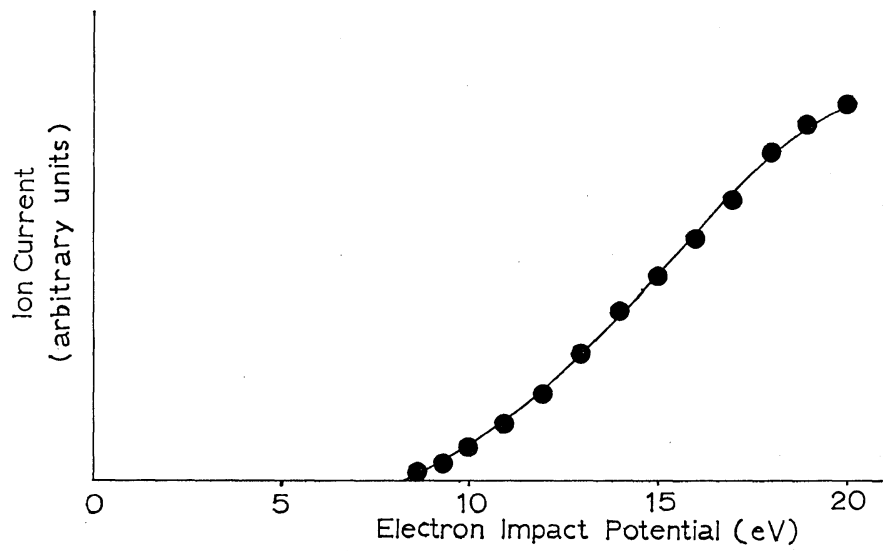

Figure 4. The relationship between ion current and electron impact potential. 


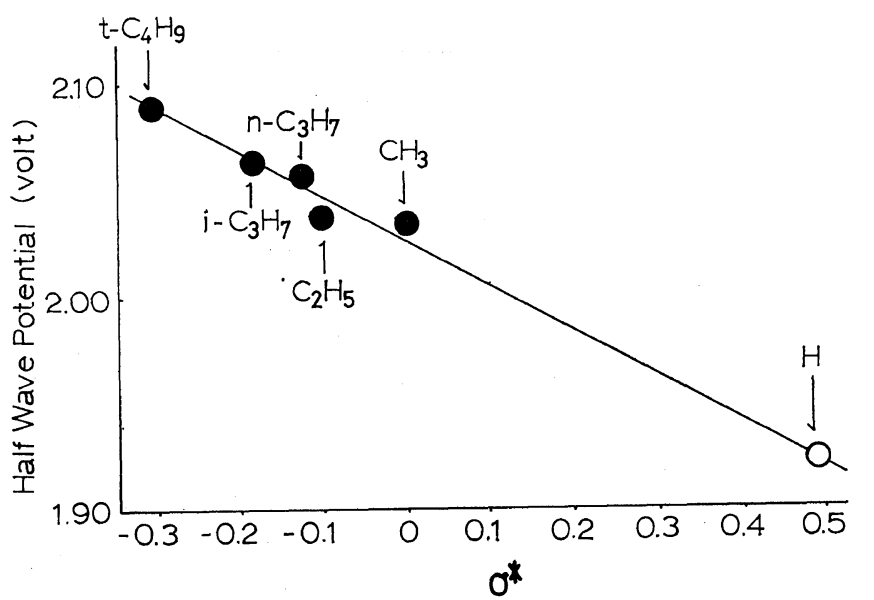

Figure 5. Taft plot of half wave potential of $\alpha, \omega$-dialkylaldehyde azine $(\mathrm{R}-\mathrm{CH}=\mathrm{N}-\mathrm{N}=\mathrm{CH}-\mathrm{R})$.

was compared with its value in the literature ${ }^{11}$ (Table III). It seems reasonable to use the value as a relative measure.

The determination of the half wave potential was attempted for use as a measure of the electron affinity. It could not be directly measured because of the polymerizability under the usual conditions of the measurement. Since a good linear relationship was found in the Taft's plot of the half wave potentials of many compounds, ${ }^{19}$ it was presumed here in the extraporation of the Taft's plot $^{12}$ for various $\alpha, \omega$-dialkylaldazines, $(\mathrm{R}-\mathrm{CH}=\mathrm{N}-\mathrm{N}=\mathrm{CH}-\mathrm{R})$, as shown in Figure 5. The value was estimated at $1.91 \mathrm{~V}$.

\section{DISCUSSION}

Numerous papers have appeared reporting the nature of Schiff bases. ${ }^{13,14}$ The carbon-nitrogen double bond tends to be more polar than an olefin bond. It is also interesting to compare the $\pi$-electrons delocalization of such symmetrical conjugated polar double bonds as formaldazine with that of the nonpolarized diene compound. This is also important for an understanding of the chemical reactivity and polymerizability of azine compounds. The $\pi-\pi^{*}$ transition of the formaldazine is assigned to $203 \mathrm{~nm}$. This wavelength is shorter than that of butadiene $(213 \mathrm{~nm})$. This suggests that the $\pi$-electrons of formaldazine are less delocalized than those of butadiene. If this is true, the torsional energy barrier around the central single bond of formaldazine will be lower than that of butadiene. This barrier was calculated by the method proposed by Fateley, et $a l^{9}{ }^{9}$ The results are shown in Table I along with some data of Fateley. ${ }^{9}$

Table I. The energy barrier of torsion around the central single bond of formaldazine and its analogues

\begin{tabular}{lc}
\hline \multicolumn{1}{c}{ Compounds } & $\begin{array}{c}\text { Energy barrier, } \\
\mathrm{kcal} / \mathrm{mol}\end{array}$ \\
\hline Formaldazine & $10.3^{\mathrm{a}}$ \\
Butadiene & $28.1^{\mathrm{a}}$ \\
Acrolein & $21.6^{\mathrm{a}}$ \\
Methyl vinyl ketone & $15.7^{\mathrm{a}}$ \\
Glyoxal & $13.7^{\mathrm{a}}$ \\
Biacetyl & $10.1^{\mathrm{a}}$ \\
Formamide & $18.3^{\mathrm{b}}$ \\
\hline
\end{tabular}

a Ref 9.

b B. Sunners, L. H. Piette, and W. Schneider, Can. J. Chem., 38, 681 (1960).

The comparison of the torsional barrier of formaldazine with those of various compounds suggests that the delocalizability of the $\pi$ electrons of formaldazine is less than that of butadiene and acrolein and is similar to that of glyoxal and methyl vinyl ketone (Table I). ${ }^{15}$ These facts show that the $\pi$-delocalization stabilization in the conjugated polar double bonds is 
much less than that of the conjugated nonpolar double bonds. These results are also consistent with the results of delocalization energy and bond order calculated by the Hückel molecular orbital theory. The parameter used in the Hückel method for the $\pi$-electron system was chosen from Yonezawa's book. ${ }^{18}$ The delocalization energy of formaldazine $(0.36 \beta)$ is smaller than that of butadiene $(0.47 \beta)$, indicating less resonance stabilization in formaldazine (Figure 7).

This was further confirmed by the bond cleavage under electron impact. High peaks in the mass spectra of formaldazine appeared at $\mathrm{m} / \mathrm{e} 56$ and 28 , which are due to the parent ion and $\left(\mathrm{CH}_{2}=\right.$ $\mathrm{N})^{+}$respectively, but the peak at $m / e$ $\left(\mathrm{CH}_{2}=\mathrm{N}-\mathrm{N}\right)^{+}$was very small. On the other hand, high peaks in the mass spectra of butadiene emerged at $m / e 54$ and 42 , which are due to the parent ion and $\left(\mathrm{CH}_{2}=\mathrm{CH}-\mathrm{CH}\right)^{+}$respectively, but the peak at $m / e 28$ due to $\left(\mathrm{CH}_{2}=\mathrm{CH}\right)^{+}$is smaller than those, as shown in Table II. The predominant bond cleavage of formaldazine occurs at the central single bond, while that of

Table II. The comparison of fragments of formaldazine and butadiene using mass spectroscopy

\begin{tabular}{|c|c|c|c|}
\hline \multirow{2}{*}{\multicolumn{2}{|c|}{$\begin{array}{c}\begin{array}{c}\text { Formaldazine } \\
M_{w}=56\end{array} \\
\mathrm{CH}_{2} \neq \mathrm{N}+\mathrm{N}=\mathrm{CH}_{2}\end{array}$}} & \multicolumn{2}{|c|}{$\begin{array}{c}\text { Butadiene } \\
M_{w}=54\end{array}$} \\
\hline & & \multicolumn{2}{|c|}{$\mathrm{CH}_{2} \neq \mathrm{CH}+\mathrm{CH}=\mathrm{CH}_{2}$} \\
\hline \multirow[t]{2}{*}{$m / e$} & $\begin{array}{l}\text { Relative } \\
\text { intensity }\end{array}$ & $m / e$ & $\begin{array}{l}\text { Relative } \\
\text { intensity }\end{array}$ \\
\hline & & 26 & 2.1 \\
\hline 27 & 4.4 & 27 & 23.3 \\
\hline 28 & 68.0 & 28 & 23.3 \\
\hline \multirow[t]{4}{*}{29} & 4.8 & & \\
\hline & & 39 & 0.8 \\
\hline & & 40 & 71.9 \\
\hline & & 41 & 2.1 \\
\hline \multirow[t]{6}{*}{42} & 2.9 & 42 & 1.7 \\
\hline & & 50 & 2.6 \\
\hline & & 51 & 7.8 \\
\hline & & 52 & 6.9 \\
\hline & & 53 & 48.5 \\
\hline & & 54 & 100.0 \\
\hline 55 & 4.8 & 55 & 3.8 \\
\hline 56 & 100.0 & & \\
\hline 57 & 2.9 & & \\
\hline
\end{tabular}

Impact potential, $20 \mathrm{eV}$. butadiene, having a higher $\pi$-delocalization stabilization, occurs at the double bond (see Table II). Various factors have been considered as the causes of this difference, ${ }^{16}$ the decrease of $\pi$ delocalization stabilization in formaldazine is likely to be one of the most important factors in the cleavage of the central single bond.

The estimated value of the ionization potential of formaldazine is shown in Table III along with

Table III. Ionization potentials of various compounds

\begin{tabular}{lcc}
\hline \multicolumn{1}{c}{ Compounds } & $\begin{array}{c}\text { Obsd } \\
\text { values, } \\
\mathrm{eV}\end{array}$ & $\begin{array}{c}\text { Ref } \\
\text { values, } \\
\mathrm{eV}\end{array}$ \\
\hline Formaldazine & 8.2 & \\
Triethylamine & 8.9 & 9.14 \\
Diethylamine & & 9.56 \\
Aniline & & 8.23 \\
1,2-Dimethylhydrazine & & 7.75 \\
Pyridine & & 9.76 \\
Butadiene & & 9.18 \\
\hline
\end{tabular}

a Ref 11

the values of similar compounds in the literature. ${ }^{11}$ The ionization potential of formaldazine is less than that of butadiene and triethylamine, and is larger than that of hydrazine. However, it must be determined whether this ionization potential is due to removing an electron from the $\pi$-orbital or the lone pair on the nitrogen atom. A radical-ion salt was synthesized by the reaction of formaldazine with chloranil and its ESR spectrum was measured at liquid nitrogen temperature. The result is shown in Figure 6. The strong single line due to the stable chloranil anion-radical; five weak lines due to the unstable formaldazine cation-radical were observed at the same $g$ value. The lines are reasonably assigned to come from the coupling of an unpaired electron with the nitrogen nuclei, because the splitting due to the coupling with hydrogen and nitrogen makes the spectrum more complicated. So, this cation radical may be produced, not by the transier of the $\pi$-electron, but by the transfer of the lone-pair electron. This consideration and the small $\pi$-delocalization stabilization of formaldazine suggests that its ionization potential is due to removing an electron 


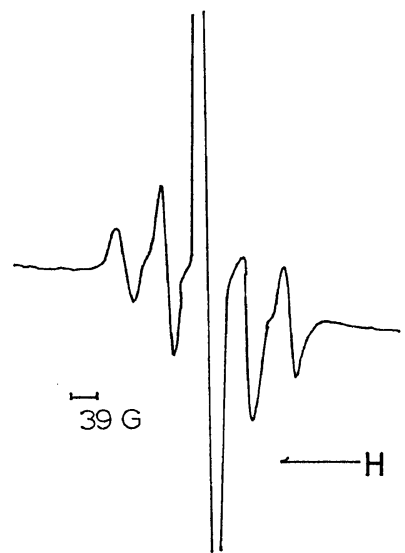

Figure 6. ESR spectrum of the complex between formaldazine and chloranil at $-78^{\circ} \mathrm{C}$.

from the lone-pair electrons. The fact that this value is larger than that of hydrazine and smaller than that of triethylamine confirms this assignment.

The half-wave potential was estimated for use as a measure of the electron affinity. The electron affinity of formaldazine is probably larger than that of acrylonitrile $\left(-E_{1 / 2} 2.01\right)$ and butadiene $\left(-E_{1 / 2} 2.60\right)$. This may be due to the higher electron affinity of nitrogen atoms in the molecule. This fact is consistent with its high anionic polymerizability.

These results can be correlated with the Hückel molecular orbital investigation. The energy level and molecular diagrams of formaldazine and butadiene are shown in Figure 7.

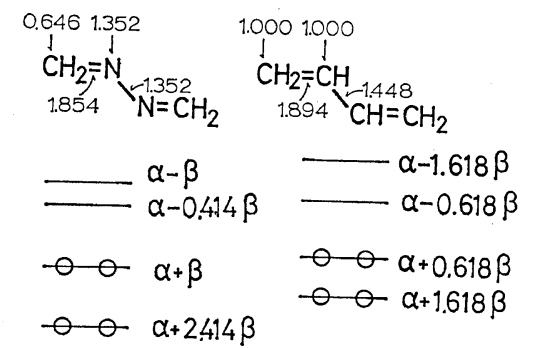

Figure 7. Molecular diagram of formaldazine and butadiene: $\alpha_{\mathrm{N}}=\alpha+\beta, \beta_{\mathrm{N}-\mathrm{N}}=\beta_{\mathrm{C}-\mathrm{N}}=\beta$.

The energy difference between the lowest vacant orbital and the highest occupied orbital of formaldazine is larger than that of butadiene. This is consistent with the results of the $\pi-\pi^{*}$ transition in the UV spectra. The bond order of the central single bond and resonant energy are smaller in formaldazine than in butadiene. The decrease of the torsional energy barrier around the central single bond is thus explained quantumchemically.

A lower energy level for the lowest vacant orbital in formaldazine than that in butadiene is consistent with the lower half-wave potential in formaldazine and the resulting higher anionic polymerizability.

In conclusion, the $\pi$-conjugation stabilization of formaldazine is smaller than that of butadiene. Formaldazine is likely to lose an electron from the lone pair, a tendency similar to that of triethylamine. Formaldazine also tends to accept an electron into its $\pi$-orbital, to a larger degree than that of acrylonitrile.

Acknowledgment. The authers are grateful to Professors S. Nozakura and K. Kuwata for several comments, and Mr. S. Ishikawa for the mesurement of far-infrared spectra.

\section{REFERENCES}

1. N. P. Neureiter, J. Amer. Chem. Soc., 81, 2910 (1959).

2. J. F. Ogilvie, Chem. Commun., 359 (1965).

3. J. F. Ogilvie and D. G. Horne, J. Chem. Phys., 48, 2248 (1968).

4. M. Kamachi and S. Murahashi, Polymer J., 6, 302 (1974).

5. M. Kamachi and S. Murahashi, unpublished work.

6. T. Curtius and E. Zinkeisen, J. Prakt. Chem., 58, 310 (1898).

7. J. Campbell, J. Amer. Chem. Soc., 59, 1980 (1937).

8. A. Weissberger, E. S. Proskauer, J. A. Riddick, and E. E. Toops, Jr., "Organic Solvents" 2nd ed, Interscience Publishers, Inc., New York, N. Y., 1955, p 704 and p 706.

9. W. G. Fateley, R. K. Harris, F. A. Miller, and R. E. Witkowski, Spectrochim. Acta, 21, 231 (1965).

10. H. H. Jaffe and M. Orchin, "Theory and Application of Ultraviolet Spectroscopy," John Wiley \& Sons, Inc., New York, N. Y., 1964, p 186.

11. R. I. Reed, "Ion Production of Electron Impact," Academic Press, New York, N. Y., 1962, p 8. 


\section{Polymerization of Formaldazine}

12. M. S. Newman, "Steric Effects in Organic Chemistry"' John Wiley \& Sons, Inc., New York, N. Y., 1956, p 591.

13. P A.S. Smith, "The Chemistry of Open-chain Organic Nitrogen Compounds,'” Benjamin, New Yerk, N. Y., 1966, p 153.

14. P. Y. Sollenberger and R. B. Martin, "The Chemistry of the Amino Group,"' S. Patai Ed., Interscience Publishers, Inc., New York, N. Y., 1968 , p 349.

15. B. Sunners, L. H. Piette, and W. Schneider, Can. J. Chem., 38, 631 (1960).

16. M. Krauss and V.H. Dibeler, "Mass Spectro- metry of Organic Ions," F. W. McLafferty, Ed., Academic Press, New York, N. Y., 1963, p 117.

17. J. D. Roberts, "Note of Molecular Orbital Calculations," Benjamin, New York, N. Y., 1962, p 81.

18. T. Yonezawa, C. Nagata, H. Kato, A. Imamura, and K. Morokuma, "Ryoshikagaku Nyumon," Kagaku Dojin, Kyoto, 1968, p 53.

19. P. Zuman, "The Elucidation of Organic Electrode Processes," Academic Press, New York, N. Y., 1969, p 112, p 152. 